\title{
Genetics evaluation for the etiologic diagnosis of autism spectrum disorders
}

\author{
G. Bradley Schaefer, $M D,{ }^{1}$ and Nancy J. Mendelsohn, $M D^{2}$
}

\begin{abstract}
Over the past decade, the reported incidence of autism spectrum disorders has continued to increase. Coincident with this, the number of referrals to clinical geneticists to identify the etiology has also dramatically increased. The reported diagnostic yield for autism spectrum disorders is commonly reported in the range of 6-15\%. However, continued advances in genetic technology expand the diagnostic options available for these evaluations and presumably increase the diagnostic yield. The list of genetic and metabolic conditions that have been reported with an autism phenotype is quite extensive. In deciding on an evaluation plan, the clinical geneticist has the difficult task of balancing an ever-expanding list of available tests and possible diagnoses with the issues of cost, practicality, and expected yield. In this article, we discuss a strategy of a tiered evaluation of the etiology of autism. These recommendations use evidence-based conclusions from the current available literature and cumulative clinical experience. Genet Med 2008:10(1):4-12.
\end{abstract}

Key Words: pervasive developmental disorders, tiered evaluations, clinical genetics

Autism was first described in 1943 by Leo Kanner. ${ }^{1}$ It is a developmental disorder of brain function with origins in prenatal life. The major features of autism are impairment of reciprocal social interactions, impairment of verbal and nonverbal communications, restricted educational activities, abnormal interests, and stereotypic behaviors. ${ }^{2}$ Infants with autism are often described as colicky or fussy babies. However, they typically come to medical attention as toddlers when differences in communication become apparent. Approximately $25 \%$ of children who fit the diagnostic criteria for autistic disorder at 2 or 3 years of age later begin to talk and communicate. By 6 or 7 years they may transition into the regular school population. For this group, social impairments usually continue. The remaining $75 \%$ have improvement with age but continue to require significant support from the parents and the school. ${ }^{3}$

Autism spectrum disorder (ASD) is an umbrella term that encompasses several conditions that share in common primary abnormalities of socialization and communication. There are five ASDs described under the diagnostic category of pervasive developmental disorders (PDD) that appear in the Diagnostic and Statistical Manual of Mental Disorders (DSM-IV) of the American Psychiatric Association. ${ }^{2}$ The five conditions are as

\footnotetext{
From the ${ }^{I}$ University of Nebraska Medical Center, Munroe-Meyer Institute for Genetics and Rehabilitation, Omaha, NE; and 'Division of Medical Genetics, Children's Hospitals and Clinics of Minnesota, Minneapolis, MN.

G. Bradley Schaefer, MD, 985430 Nebraska Medical Center, Omaha, NE 68198-5430. E-mail: gbschaef@unmc.edu.

Disclosure: The authors declare no conflict of interest.

Submitted for publication August 15, 2007.

Accepted for publication September 19, 2007.

DOI: $10.1097 /$ GIM.0b013e31815efdd7
}

follows: (1) autistic disorder-sometimes called "classic" autism - is the most commonly identified type of ASD, (2) Asperger syndrome, (3) pervasive developmental disorder-not otherwise specified (PDD-NOS), (4) childhood disintegrative disorder, and (5) Rett syndrome.

The diagnosis of ASDs is still a clinical one. It is based on behavioral history and behavioral assessments that document persistent deficits in reciprocal social behavior, communication, and restricted interests or repetitive behavior. ASDs should be diagnosed by trained professionals using objective criteria. In recent years, the use of internationally available and validated diagnostic tools has led to greater consistency in diagnoses. ${ }^{4,5}$

The reported prevalence of autism has increased over the past 10 years with estimates for autism now being on the order of 10-60 per 10,000 individuals, if all forms of ASD are considered. ${ }^{6}$ In fact, the Center for Disease Control and Prevention has recently estimated the prevalence of ASDs in the United States as approximately 5.6 per 1000 children. ${ }^{7}$ The rise in the reported prevalence of ASD may in part be due to better knowledge of the disease variability, broader diagnosis with improved public, and a higher degree of professional awareness of the disorder.

Epidemiologic data support the presumption that autism is primarily a genetic disorder. The calculated heritability of autism is around $90 \% .^{8-11}$ ASDs occur four times as often in men as in women, with an even higher ratio in the milder forms. Concordance in twins is high. There is a 70\% concordance in monozygotic twins; $90 \%$ if a broader definition of ASD is used. ASDs show a significant familial recurrence rate much higher than expected given the general population occurrence. The generally reported recurrence risk is $4 \%$ if the first affected child is female and $7 \%$ if first child is male. In reality, the 
occurrence of second affected child is around $2-3 \%$ presumably due to a reduced number of families having a subsequent child after having a child with autism. The recurrence risk increases even more with a second affected child up to 50\%.12-16 Thus the familial pattern in autism seems very consistent with multifactorial inheritance.

The diagnosis of ASD is a wide encompassing disorder that does not imply etiology. Appropriate management of all persons diagnosed with an ASD includes a comprehensive genetics evaluation. The genetics evaluation attempts to define the etiology by a careful diagnostic evaluation. Families should be informed before undertaking such an evaluation, the potential yield, and the benefits of this process.

The generally reported range for identifying a diagnosis in persons with autism is $6-15 \%$. Recent reports of the yield of a genetics diagnostic evaluation for autism range from $10 \%{ }^{17}$ to $40 \%{ }^{18}$ Abdul-Rahman and Hudgins ${ }^{19}$ reviewed the evaluation of 101 patients referred with the diagnoses of PDD-NOS, atypical autism, Asperger syndrome, or autistic features, which did not necessarily meet the DSM-IV criteria for PDD. The yield $(8.3 \%)$ in this "nonclassic" group was similar to that reported in classic autism. The majority of the cases identified in this review were chromosomal abnormalities and MECP2 mutations. Regardless of the study, in general, the diagnostic yield seems to increase with the extent of the evaluation.

\section{RATIONALE/EVIDENCE}

As mentioned, the epidemiology of the ASDs clearly suggests multifactorial inheritance. The emerging themes in multifactorial conditions are (1) genetic heterogeneity with multiple major gene effects, (2) physiologically linked processes with multiple genes at work within each process, and (3) typically modest environmental buffering. ${ }^{12,14,16,20,21}$ All of these factors are clearly applicable to autism.

Basic research into the multiple etiologies of autism progresses at a rapid pace. It is the task of the clinical geneticist to assimilate this continually changing information base and decide when sufficient evidence exists to incorporate new information into clinical practice. A key component of this decision-making process is to decide when the reported association of a particular genetic condition is "strong" enough to justify testing for the condition in an autism evaluation. It is the purpose of this article to provide evidences and rationale behind such decisions.

The role of the clinician is to try and identify specific etiologic (unifactorial) conditions and then provide diagnosisspecific counseling. Clearly, the work up must be customized to the individual patient, taking into consideration focused information from the history and physical and clinical experiences. There is no single approach or algorithm that can be applied to all cases. Still, generalizations can be made and templates used as a starting point for structuring these evaluations. In the evaluation of genetically complex conditions, several basic concepts must be followed for a successful outcome. For many practical reasons, a stepwise (tiered) evaluation is the preferred approach-rather than performing a "shotgun" evaluation in which dozens of tests are ordered as a matter of routine. The evaluations should be structured so that tests obtained in higher tiers have a higher expected diagnostic yield, lower invasiveness of testing, better potential of intervention, and easier overall practicality of obtaining the tests. In our experience, this approach has a high level of acceptance with third-party payers and with families. ${ }^{18}$

The first, and potentially most important, step in the diagnostic evaluation of ASDs is the pre-evaluation. Several pieces of critical information need to be obtained before beginning any investigation. Obviously, an accurate diagnosis is necessary. Before evaluating the patient for a genetic cause of autism, it should be confirmed that the diagnosis is indeed correct for the patient. The diagnosis should be made by a professional trained in the area of autism diagnoses using strict criteria and appropriate objective tools. ${ }^{22}$ All patients diagnosed with autism should have a formal audiogram. Clinical experience and the medical literature consistently identify patients with a hearing loss mistakenly diagnosed as having autism. ${ }^{18,23}$ Problems with hearing would obviously alter socialization and communication. It is imperative that normal hearing be confirmed before settling upon a diagnosis of autism. In addition, the results of cognitive testing should be known and incorporated into the diagnostic work up. It is not surprising that more significant degrees of cognitive impairment are associated with greater impairments of socialization and communication (autistic symptoms). It is important that the autistic-like symptoms of severe cognitive impairments not be confused with a primary disorder of socialization and communication. There is debate as to the role of obtaining an electroencephalogram in the evaluation of autism. Persons with Landau-Kleffner syndrome have an acquired aphasia associated with an epileptiform disorder. There are reports of persons with Landau-Kleffner syndrome carrying the diagnosis of autism with the aphasia being deemed a primary disorder of communication. ${ }^{24}$ This is a particularly attractive cause of autism for families because it has a straightforward medical therapy. In general, we would suggest that an electroencephalogram does not need to be performed in all persons with autism, but only in those cases where the clinical history suggests a possible seizure disorder. Finally, information about any prior evaluations should be obtained. A recent policy statement by the American Academy of Pediatrics ${ }^{25}$ recommends that the primary care provider for a person with autism could/should initiate the diagnostic work up. This document specifically recommends obtaining a highresolution karyotype and Fragile X studies as the initial step. Clearly, the consulting geneticist should be aware of any such prior testing before constructing a diagnostic plan.

Another important issue in the pre-evaluation component of the work up is deciding which patients warrant an evaluation. There is a long list of genetic syndromes that have been reported with an association with autism/autistic behaviors. ${ }^{26}$ For many of these conditions (e.g., Fragile X syndrome, Smith-Lemli-Opitz syndrome) the association is so strong that the primary diagnosis may be confidently assigned as the 
Table 1

Partial list of genetic syndromes with a reported association with autism

\begin{tabular}{ll} 
No work up indicated & \multicolumn{1}{c}{$\begin{array}{c}\text { Autism evaluation } \\
\text { indicated }\end{array}$} \\
\hline Fragile X syndrome & Apert syndrome \\
Rett syndrome & Williams syndrome \\
Angelman syndrome & \\
De Lange syndrome & \\
Prader-Willi syndrome & Noonan syndrome \\
Smith-Lemli-Opitz syndrome & Down syndrome \\
Smith-Magenis syndrome & Turner syndrome \\
Tuberous sclerosis & Neurofibromatosis \\
CHARGE syndrome & Myotonic dystrophy, \\
& Duchenne dystrophy \\
Shprintzen syndrome (22q11 deletions) & Moebius anomalad \\
Sotos syndrome & Cohen syndrome \\
PTEN associated disorders (Cowden syndrome, & Oculo-auriculo-vertebral \\
Bannayan-Riley-Ruvalcaba syndrome) & spectrum \\
Hypomelanosis of Ito & Joubert syndrome \\
Lujan-Fryns syndrome & \\
\hline
\end{tabular}

etiology of autism in the patient-and hence further investigation into the cause of autism is not necessary. For others, an association has been reported, but the association is not as strong or is still debated. For these, there does not seem to be sufficient evidence to confidently identify the condition as the cause of autism in the patient and hence a diagnostic evaluation would seem prudent. Table 1 contains a partial list of conditions associated with autism and our recommendations as to when an evaluation seems indicated.

For the remainder of our discussions, we will limit ourselves to thoughts and recommendations for the clinical genetic evaluation of idiopathic (nonsyndromic, uncomplicated) autism. In this regard, the concept of an "expanded phenotype" should be mentioned. Ranges of phenotypes have been described for many specific genes. Typically, a particular locus is associated with a known genetic syndrome. Once a genotype:phenotype relationship has been established, it is not uncommon for other phenotypes to be reported in association with changes in the same gene. Ultimately a broad spectrum of phenotypes may be reported in association with that particular gene. Often, this range encompasses both syndromic and nonsyndromic phenotypes. Rett syndrome is a prime example. Not long after MECP2 was identified as the gene primarily responsible for the Rett syndrome phenotype, other patients were reported with a myriad of other clinical presentations due to $M E C P 2$ mutations. With this understanding, MECP2 gene testing is now indicated in the diagnostic work up of nonsyndromic mental retardation, cerebral palsy, and autism. ${ }^{27}$ Thus, the diagnostic testing for patients with autism will include testing for specific genetic changes that are not limited in their expression to an autism phenotype.

\section{Conditions identified as possible causes of idiopathic autism}

1. Cytogenetic changes are consistently reported as one of the most common identifiable causes of autism. The reported diagnostic yield for prometaphase chromosome studies is around 3-5\%. ${ }^{16,28-30}$ There is little debate that a high-resolution karyotype should be performed on all individuals with autism. The literature contains reports of innumerable cytogenetic changes reported in autism. The most commonly reported (recurrent) cytogenetic abnormalities found in persons with autism are deletions involving 7q, 22q13, 2q37, 18q, and $\mathrm{Xp}$ and sex chromosome aneuploidies (47,XYY and 45,X/ 46,XY mosaicism). ${ }^{28,30-32}$ Neurocutaneous conditions such as Hypomelanosis of Ito have also been associated with autism. ${ }^{33,34}$ A skin biopsy with a fibroblast karyotype should be considered in patients with pigmentary abnormalities and a normal leukocyte karyotype. Recent evidence suggests that microarray-based comparative genomic hybridization (aCGH) may be a highly effective tool in detecting somatic chromosomal mosaicism. ${ }^{35}$ If these early findings are confirmed, such studies could replace fibroblast studies as a less invasive alternative in the future.

The advent of fluorescent in situ hybridization (FISH) techniques has tremendously enhanced the diagnostic capabilities of clinical geneticists in almost every part of their clinical scope of practice. Application of FISH technology to the diagnostic work up of autism is a relatively recent-and often debatedaddition. Of course, individual FISH studies would be indicated in the confirmation of a clinically suspected condition (e.g., Smith-Magenis syndrome).

By and large, FISH studies have confirmed and expanded the list of chromosomal "hot spots" in autism (Table 2). In general, the subtelomeric FISH panel has not been shown to be a high-yield test in autism. ${ }^{36-39}$ However, it could be argued that such testing is absolutely indicated in patients with an IQ $<50^{40}$, which would be a common comorbidity in patients with autism. Regardless, the development of aCGH has prob-

Table 2

Chromosomal "Hot Spots" for autism spectrum disorders

Most common
$15 \mathrm{q} 11-13$
$7 \mathrm{q} 22-31$
$22 \mathrm{q} 11$
$22 \mathrm{q} 13$
$2 \mathrm{q} 37$
Others
Xp
$18 \mathrm{q}$
$17 \mathrm{p}$
$17 \mathrm{q}$
$16 \mathrm{p}$


ably rendered this argument void. Depending on the construction of the platform, aCGH can include the probes for the 41 subtelomeric regions, probes for regions highly associated with autism (Table 2), and probes for selected loci in which the expanded phenotypes are commonly associated with autism. Given a choice, a platform that includes probes for the $15 \mathrm{q}$ pericentromeric region, $17 \mathrm{p} 11,22 \mathrm{q} 11,22 \mathrm{q} 13,2 \mathrm{q} 37$, and the subtelomeric regions would seem appropriate for an autism evaluation. A recent report by Jacquemont et al. ${ }^{41}$ would seem to support this conclusion. In this study, the authors found aCGH detectable abnormalities in 8 of 29 (27.5\%) patients with syndromic (complex) autism. We have found similar results in our clinical experience. ${ }^{42}$ Although some might opine that it is still too early to universally recommend aCGH in the evaluation of autism, we feel that the potential diagnostic yield, coverage of a host of important loci, and the relatively low cost, warrant having aCGH as a standard part of an autism evaluation.

2. The association of Fragile $X$ syndrome with autism is well documented. The incidence of appropriately diagnosed autism patients with Fragile $\mathrm{X}$ syndrome (full mutation) is around 15-20\%. ${ }^{43-45}$ Conversely, a fully expanded trinucleotide sequence in the FMR-1 gene has been reported in $3-10 \%$ of unselected patients with autism. ${ }^{44,46-48}$ Clearly, sufficient evidence exists to recommend Fragile X testing in an unselected population of patients with autism.

3. As mentioned earlier, mutations in the MECP2 gene have been associated with a nonsyndromic autism phenotype. To date, we are not aware of any reported males with idiopathic autism and MECP2 mutations. However, MECP2 mutations have been reported relatively frequently $(3-13 \%)$ in females with autism. ${ }^{19,49}$ Thus, MECP2 gene sequencing should be considered in the earlier stages of the evaluation of females with autism.

4. The association between a large head size (both macrocephaly and macroencephaly) and autism has been consistently noted. A head circumference greater than the 50th percentile has been reported in $83 \%$ of patients with autism, and $24 \%$ of patients have a head circumference over the 98th percentile. ${ }^{50-53}$ The exact basis of this association has not been delineated to date. In addition, recent reports have demonstrated a surprisingly high frequency of PTEN mutations in patients with idiopathic autism. In an unselected cohort, PTEN mutations were found in 2 of 71 (3\%) patients. ${ }^{17}$ Notably, both patients were macrocephalic. In another study, ${ }^{54}$ PTEN mutations were seen in 3 of 18 (17\%) patients selected with autism and macrocephaly-defined as a head circumference $>2.5$ standard deviations above the mean. Thus, PTEN gene sequencing should be considered in all patients with autism in which the head circumference is $>2.5$ standard deviations greater than the mean.

5. It has been reported that essentially $100 \%$ of patients with Smith-Lemli-Opitz syndrome have autism. ${ }^{55}$ A recent study by Tierney et al. ${ }^{56}$ evaluated 100 children with autism and at least one affected relative (with the hopes of increasing the yield for single gene etiologies). No cholesterol me- tabolite abnormalities were identified, but almost 20\% had low total serum cholesterol levels. To our knowledge, no studies to date have been performed in which 7-dehydrocholesterol studies have been performed as part of routine testing in an unselected large cohort of individuals with idiopathic autism. Thus, the diagnostic yield for this test cannot be currently estimated. Although it would be our inclination to perform these studies, given our suspicion that this may account for a significant number of cases, we cannot support this as a standard part of a tiered evaluation without some reservation.

6. A host of single genes have been identified in association with autism. These loci have been identified by a variety of techniques including whole genome searches, linkage studies, and candidate gene analysis. Currently, very few of these genes can be analyzed by clinically available testing. Two such genes bear mentioning. Neuroligin 3 (NGN3) and neuroligin 4 (NGN4) are $\mathrm{X}$-linked genes that have been associated with an autism phenotype. Clinical testing for these two genes is availablemost commonly as a component of an X-linked mental retardation gene panel. ${ }^{57}$ Again, routine testing for changes in these genes has not been reported in cohorts of patients with autism. Although testing is available clinically, we would suggest using these tests only for those cases of autism where a clear X-linked pattern of neurobehavioral/neurodevelopmental disorders and cognitive deficits can be ascertained in the patient and his/her family.

7. The association of certain metabolic disorders with autism has been known for some time. Patients with untreated phenylketonuria are consistently reported to have distinct autistic behaviors. ${ }^{58}$ With current newborn screening practices in place in the United States, this is hoped to be of historical interest only. The early neurologic features of mucopolysaccharidoses may look strikingly similar to what is seen in the so-called autistic regression syndrome. Although some would strongly advocate routine metabolic testing in patients with autism, there is little evidence that "standard" metabolic testing is a high-yield diagnostic option in patients with autism without independent clinical indicators of a metabolic disorder. With the addition of expanded newborn screening using tandem mass spectroscopy, many such conditions will have been screened for as a newborn. A very low-cost component of the evaluation would be to simply obtain the results of the patient's newborn screening. Recent years have seen the description of a host of newly described metabolic conditions that have been reported in association with an isolated autism phenotype (Table 3 ). Testing for these conditions can be quite involved and require the utilization of multiple reference laboratories across continents. As with other conditions noted earlier, these have been mainly reported anecdotally, and have not been assessed as part of a systematic study. As such, routine testing for these disorders cannot be recommended at this time. In the meantime, targeted screening would seem to be a reasonable compromise. Screening would entail random serum and urine uric acid levels. If 
Table 3

Metabolic conditions reported in association with an isolated autism phenotype

Disorders of purine metabolism

Adenylosuccinate lyase (ADSL) deficiency

Phosphoribosylpyrophosphate (PRPP) synthetase superactivity

Disorders of pyrimidine metabolism

Dihydropyrimidinase (DPD) deficiency

Cytosolic 5' nucleotidase (NT) superactivity

Unknown sulfation defect

Urine $S$-sulfocysteine

Disorders of GABA metabolism

Succinic semialdehyde dehydrogenase (SSADH) deficiency

Disorders of creatine metabolism

Guanidinoacetate methyltransferse (GAMT) deficiency

$\mathrm{X}$-linked creatine transporter defect

Conditions that infer susceptibility to autism phenotype

Monoamine oxidase A (MOA)

Glyoxylase A (GLYA)

these are elevated, obtain a hypoxanthine-guanine phosphoribosyl transferase and phosphoribosylpyrophosphate synthetase superactivity testing. Alternatively, if they are low, obtain a purine/pyrimidine panel (uracil excretion plus xanthine and hypoxanthine levels).

8. Teratogenic causes have often been implicated in autism. To date, fetal rubella syndrome seems to be the most convincing. ${ }^{59}$ Other teratogens that have been reported with autism, but with less support in the literature include cytomegalovirus, ethanol, thalidomide, and valproic acid. ${ }^{60-62}$ Clearly, an important component of the evaluation includes a careful prenatal history with targeted questioning about potential teratogenic exposures.

9. Probably the greatest debate that exists regarding an autism evaluation concerns the role of neuroimaging. In studies in which neuroimaging is performed as a standard component of an autism evaluation, the yield can be quite significant. ${ }^{18}$ Intuitively, it seems reasonable to "examine" the organ that is dysfunctional. It is thus not surprising that imaging the brain in patients with autism would yield positive findings on brain anatomy and organization. Sometimes, the neuroimaging studies will provide a definitive diagnosis, such as tuberous sclerosis with no observable external physical features. ${ }^{18}$ Often, these studies will identify subtle markers of cerebral dysgenesis. Although the identification of such markers does not provide complete insight into etiology, we still find knowledge of such anomalies very helpful to clinicians and families. Including brain imaging (with magnetic resonance imaging as the test of choice) as a "third tier" study for all patients with autism seems appropriate to us at this time. Still, we acknowledge that this view is not univer- sally accepted. We are aware that many practitioners choose an intermediate approach and perform imaging studies only in those patients with other clinical indicators (e.g., macrocephaly, microcephaly, seizures).

10. Advances in genetic technology and in the understanding of complex genetic mechanisms have identified de novo copy number mutations and small genomic rearrangements in patients with autism. ${ }^{63,64}$ The recent reports of these associations seems strong. If additional studies confirm these associations, testing for these changes may be added to the standard autism genetic evaluation.

11. Epigenetic causes of autism have already been documented in cases of Rett syndrome (MECP2 mutations) and Angelman syndrome. It is likely that other epigenetic causes and/or modifiers will be identified. ${ }^{65,66}$

\section{Suggested (model) approach}

Incorporating all the above information, we have constructed a model evaluation scheme for the clinical genetic approach to defining an etiologic basis in autism. This scheme is summarized in Table 4. As emphasized earlier, this scheme may serve as a template, but each evaluation requires the geneticist to use his/her judgment and experience in refining the plan. The reader is referred to the first part of this section for a detailed discussion of the "pre-evaluation" component of the work up.

Once the pre-evaluation process is complete, the first tier of the process can begin. At the time of the initial consultation, the geneticist should obtain a detailed history and perform a dysmorphology examination. The major components of this are similar to the approach to other genetic conditions. Table 5 provides a list of important information to include when obtaining the history, and Table 6 lists important clues on the examination. Given earlier discussion on the importance of pigmentary changes, the examination should always include a Woods lamp screening component. Prometaphase chromosomes and Fragile X testing should be accomplished if not already performed before the referral. "Standard" metabolic screening and rubella titers should be performed only if clinical indicators are present. The results of the patient's newborn screening should be requested and reviewed.

If the first tier of the evaluation provides a definitive answer, the work up is over and definitive counseling can be provided. If the first tier evaluation does not provide an answer, the work up can then proceed to the second tier. In this next level, a fibroblast karyotype can be obtained if the leukocyte karyotype was normal and if significant pigmentary changes are seen on the initial examination. Additional laboratory testing would include aCGH, MECP2 gene testing (for females only), and $P T E N$ if the head circumference is $>2.5$ standard deviations above the mean.

In the third tier neuroimaging (preferably magnetic resonance imaging) is performed. The remainder of the evaluation would include chromosome 15 methylation testing and serum/urine uric acid levels. As discussed earlier, cholesterol/7- 
Table 4

Template for the clinical genetic diagnostic evaluation of autism spectrum disorders

Pre-evaluation

- Confirmation of diagnosis of autism by trained professional using objective criteria and tools

- Sensory screening (complete audiogram)

- Electroencephalogram (EEG) - if clinical questions

- Cognitive testing

[Prometaphase chromosomes and Fragile X studies may be performed before referral]

First tier

Initial evaluation to identify known syndromes or associated conditions

- Dysmorphology/clinical genetics

- Should include Woods lamp evaluation

- If specific diagnosis is suspected, proceed with targeted testing

- Rubella titers - if clinical indicators present

○ "Standard" metabolic screen — if clinical indicators present

- Urine mucopolysaccharides and organic acids

- Serum lactate, amino acids, ammonia, and acyl-carnitine profile

- Check results/type of newborn screen

- Prometaphase chromosomes - if not already performed

- DNA for Fragile X - if not already performed

Second tier

- Fibroblast karyotype if leukocyte karyotype is normal and significant pigmentary abnormalities are noted

- Comparative genomic hybridization (chromosomal microarray)

- MECP2 gene testing (females only)

- PTEN gene testing (if the head circumference is 2.5 standard deviations greater than the mean)

Third tier

- Brain magnetic resonance imaging (MRI)

- Chromosome 15 methylation

- Serum and urine uric acid

$\circ$ If elevated, Hypoxanthine-guanine phosphoribosyl transferase (HgPRT) and Phosphoribosylpyrophosphate (PRPP) synthetase superactivity testing

○ If low, purine/pyrimidine panel (uracil excretion, xanthine, hypoxanthine)

dehydro-cholesterol assessment could be included here, although it is hard to be dogmatic about its inclusion.

This list is by no means exhaustive. There are many other conditions that have been reported in association with autism and for which there is clinical testing. For most of these, the reported association is typically anecdotal and usually in a few random case reports. For now, these would have to be classified as further potential tests of unknown/unproven value (Table 7). It is stating the obvious to mention that what is needed is a
Table 5

Important elements on the patient history
Prenatal history

Maternal rubella status

Maternal thyroid disease

Teratogenic exposures

Cytomegalovirus

Ethanol

Thalidomide

Valproic acid

Medical history

Dystonia

Electrolyte abnormalities

Failure to thrive

Food intolerances

Hypoglycemia

Other paroxysmal disorders

Seizures

Family History

Cancer (especially thyroid), and hamartomatous neoplasms of the skin and mucosa, GI tract, bones, central nervous system, eyes, and genitourinary tract

Congenital anomalies

X-linked pattern of neurodevelopmental/neurobehavioral disorders

large multicenter collaborative study to prospectively screen for these (and the tests above) in a prospective manner in an unselected cohort of patients with idiopathic autism.

\section{SUMMARY OF RECOMMENDATIONS}

The current literature would suggest that the overall diagnostic yield for a clinical genetic evaluation into the etiology of autism is on the order of $15 \%$. A critical review of the potential contribution of incorporating newer testing techniques suggests that yield can be significantly higher. Using the above information collectively, the following yields could conservatively be projected as

- Prometaphase chromosomes (5\%)

- $\operatorname{aCGH}(10 \%)$

- Fragile X (5\%)

- MECP2 (5\% females)

- $\operatorname{PTEN}(3 \%)$

- Other (10\%).

Thus the overall diagnostic yield using standard, clinically available testing in conjunction with an astute clinical assessment is on the order of $40 \%$. This is in keeping with a recent (albeit somewhat small) retrospective study. ${ }^{18}$ 
Table 6

Important clues on physical examination

Head size

Macrocephaly — PTEN

Microcephaly — Rett syndrome, fetal rubella

Pigmentary abnormalities

Multiple findings - tuberous sclerosis complex

Penile freckling - PTEN mutations

Pigmentary variances that follow dermatome lines — chromosomal mosaicism Subtle dysmorphic features

Lip "sneer" — Smith-Magenis syndrome

Syndactyly — Smith-Lemli-Opitz

Retinal changes - fetal rubella

Important clues on medical history

Seizures and or neuroregression

MECP2, ARX mutations

Genetic syndromes — tuberous sclerosis, Angelman syndrome

Cerebral dysgenesis

Metabolic disorders

Acquired aphasia

Landau Kleffner syndrome

Table 7

Additional genetic tests/conditions reported in association with an autism phenotype: unknown/unclear utility

- FMR1 gene sequencing

- Testing for (non-FMR1) folate sensitive fragile sites

- 7-Dehydro-cholesterol

- UBE3A gene sequencing

- NSD1 sequencing/microdeletion screening

- Mitochondrial gene sequencing

- Neuroglian $(N G N)$ gene testing (may be as a component of an X-linked mental retardation gene panel)

- Extended metabolic work up (consider referral to metabolic specialist)

- Bratton and Marshall test to screen for succinyl-AICAriboside; HPLC to confirm suspected cases

$\circ$ Urinary creatine excretion

$\circ$ GC-MS of organic acid extracts in urine

○ HPLC of nucleotides in erythrocytes

○ Urine $S$-sulfocysteine

○ Urine gamma-hydroxy-butyric acid

HPLC, high pressure liquid chromatography; GC-MS, gas chromatography mass spectroscopy.

\section{Benefits}

Establishing a diagnosis and identifying the underlying etiology of ASD helps families with parental anxiety, prognosis, care models, and abilities to recognize comorbid conditions, and provides a recurrence risk to families. ${ }^{23}$ There is clear benefit for most families simply in the answer to the question of why a child has these problems. A correct etiologic diagnosis provides the family and the care providers with direction for appropriate case management and an awareness of associated medical conditions. The child with tuberous sclerosis, for example, will require a significantly different medical follow-up regimen when compared with the child with a chromosomal aneuploidy.

As advances in genetic technology become clinically available, the geneticist has the opportunity to move from empiric recurrence risk counseling to diagnosis-related counseling. The advantage, of course, is to provide the family with information specific to the etiology for their family rather than providing population-averaged data. Practitioners outside of clinical genetics often question the rationale for an aggressive diagnostic evaluation. Concerns cited include cost, difficulty with third-party payers, and the general lack of therapeutic implications in achieving an etiologic answer. While the logic for an evaluation should seem intuitive to a clinical geneticist, the rationale for proceeding with diagnostic testing may need to be reviewed with the family and with referral sources. It is our contention that all patients with autism be offered a thorough diagnostic evaluation.

\section{Limitations}

Despite all the potential benefits for the patient (family) as noted above, there are several practical limitations to proceeding with a diagnostic evaluation for patients with autism. In general, these issues are not specific to autism, and are well known to practicing geneticists.

As with any patient care activity, informed consent is critical. The patient (family) should be given detailed information about the proposed diagnostic approach, the expected cost, and the diagnostic yield. In addition, they should be informed at the beginning as to what type of information a diagnosis would or would not provide them. Referral sources should also receive comparable information.

Expense can be a significantly limiting factor. The overall estimated cost of the evaluation proposed in this article could easily approach $\$ 8000$. Clearly, questions about the "cost effectiveness" of this type of evaluation have been raised and are likely to continue. In addition, many third-party payers have specific exclusion clauses for autism. Although clinical geneticists recognize this as being no different from the evaluation of a child with mental retardation or cerebral palsy, third-party payers may not. The aura of autism being a "mental disorder" has persisted in many organizations. The understanding of autism as a true neurogenetic disorder has not permeated most levels of health care.

A practical limitation of the tiered evaluation approach is the time involved in completing all levels of testing. This requires a great deal of patience on the family's part. If the time for preauthorization of procedures is factored in, the time from the generation of the referral to the completion of a third 
tier of testing may take 6 months or longer. It is important that this be discussed with the family at the beginning of the process.

Finally, the psychosocial impact of a genetic diagnosis can never be underestimated. The clinical geneticist in cooperation with his/her genetic counselor colleague needs to be prepared to support the family throughout the entire process, which by design will be costly in time, expense, resources, and psychosocial stressors.

\section{APPENDIX}

\section{EDUCATIONAL AND INFORMATIONAL RESOURCES}

○ Autism Society of America

Publishes the "Advocate," an informative quarterly magazine.

7910 Woodmont Avenue Suite 300

Bethesda MD 20814-3067

Phone: 800-3AUTISM (800-328-8476); 301-657-0881

Fax: 301-657-0869

E-mail: Advocate@autism-society.org

www.autism-society.org

$\bigcirc$ Autistic Spectrum Disorders: Best Practice Guidelines for Screening, Diagnosis, and Assessment California Department of Developmental Services (2002) www.ddhealthinfo.org/pdf/ASDGuidelines.pdf

○ National Institute of Neurological Disorders and Stroke Autism Fact Sheet

\section{O AGRE: Autism Genetics Resources Exchange} 54455 Wilshire Blvd Suite 715

Los Angeles CA 90036

Phone: 323-549-0547

E-mail: info@agre.org

www.agre.org

○ "Understanding Autism Spectrum Disorders (ASDs)" aims to answer. The 44-page booklet, developed by the Academy's Autism Expert Panel, was written to answer the questions of parents of children who have ASD or used as a resource for health care professionals to provide to parents upon their child's diagnosis.

\section{References}

1. Kanner L. Autistic disturbances of affective contact. Nerv Child 1943;2:217-250.

2. Diagnostic and statistical manual of mental disorders (DSM-IV), 4th ed. Washington, DC: American Psychiatric Press, Inc; 1994.

3. Seltzer MM, Shattuck P, Abbeduto L, Greenberg JS. Trajectory of development in adolescents and adults with autism. Ment Retard Dev Disabil Res Rev 2004;10(4): $234-247$.

4. Lord C, Rutter M, Le Couteur A. Autism Diagnostic Interview-Revised: a revised version of a diagnostic interview for caregivers of individuals with possible pervasive developmental disorders. J Autism Dev Disord 1994;24(5):659-685.
5. Lord C, Risi S, Lambrecht L, Cook EH Jr, et al. The autism diagnostic observation schedule-generic: a standard measure of social and communication deficits associated with the spectrum of autism. J Autism Dev Disord 2000;30(3):205-223.

6. Fombonne E. The prevalence of autism. JAMA 2003;289(1):87-89.

7. Mental health in the United States: parental report of diagnosed autism in children aged 4-17 years-United States, 2003-2004. MMWR Morb Mortal Wkly Rep 2006; 55(17):481-486.

8. Bailey A, Le Couteur A, Gottesman I, Bolton P, et al. Autism as a strongly genetic disorder: evidence from a British twin study. Psychol Med 1995;25(1):63-77.

9. Piven J, Palmer P, Jacobi D, Childress D, et al. Broader autism phenotype: evidence from a family history study of multiple-incidence autism families. Am J Psychiatry 1997;154(2):185-190.

10. Chakrabarti S, Fombonne E. Pervasive developmental disorders in preschool children. JAMA 2001;285(24):3093-3099.

11. Hollander E, King A, Delaney K, Smith CJ, et al. Obsessive-compulsive behaviors in parents of multiplex autism families. Psychiatry Res 2003;117(1):11-16.

12. Cook EH Jr. Genetics of autism. Child Adolesc Psychiatr Clin N Am 2001;10(2):333350 .

13. Lotspeich LJ, Ciaranello RD. The neurobiology and genetics of infantile autism. Int Rev Neurobiol 1993;35:87-129.

14. Muhle R, Trentacoste SV, Rapin I. The genetics of autism. Pediatrics 2004;113(5): e472-e486.

15. Simonoff E. Genetic counseling in autism and pervasive developmental disorders. J Autism Dev Disord 1998;28(5):447-456.

16. Spence SJ. The genetics of autism. Semin Pediatr Neurol 2004;11(3):196-204.

17. Herman GE, Henninger N, Ratliff-Schaub K, Pastore M, et al. Genetic testing in autism: how much is enough? Genet Med 2007;9(5):268-274.

18. Schaefer GB, Lutz RE. Diagnostic yield in the clinical genetic evaluation of autism spectrum disorders. Genet Med 2006;8(9):549-556.

19. Abdul-Rahman OA, Hudgins L. The diagnostic utility of a genetics evaluation in children with pervasive developmental disorders. Genet Med 2006;8(1):50-54.

20. Chudley AE. Genetic landmarks through philately—autism spectrum disorders: a genetic update. Clin Genet 2004;65(5):352-357.

21. Fombonne E. Modern views of autism. Can J Psychiatry 2003;48(8):503-505.

22. Battaglia A. On the selection of patients with developmental delay/mental retardation and autism spectrum disorders for genetic studies. Am J Med Genet A 2007; 143(8):789-790.

23. Battaglia A, Carey JC. Diagnostic evaluation of developmental delay/mental retardation: an overview. Am J Med Genet C Semin Med Genet 2003;117(1):3-14.

24. Stefanatos GA, Kinsbourne M, Wasserstein J. Acquired epileptiform aphasia: a dimensional view of Landau-Kleffner syndrome and the relation to regressive autistic spectrum disorders. Child Neuropsychol 2002;8(3):195-228.

25. American Academy of Pediatrics: The pediatrician's role in the diagnosis and management of autistic spectrum disorder in children. Pediatrics 2001;107(5):12211226.

26. Artigas-Pallares J, Gabau-Vila E, Guitart-Feliubadalo M. [Syndromic autism. II. Genetic syndromes associated with autism.]. Rev Neurol 2005;40(Suppl 1):S151S162.

27. Erlandson A, Hagberg B. MECP2 abnormality phenotypes: clinicopathologic area with broad variability. J Child Neurol 2005;20(9):727-732.

28. Reddy KS. Cytogenetic abnormalities and fragile-X syndrome in Autism Spectrum Disorder. BMC Med Genet 2005;6:3.

29. Shevell MI, Majnemer A, Rosenbaum P, Abrahamowicz M. Etiologic yield of autistic spectrum disorders: a prospective study. J Child Neurol 2001;16(7):509-512.

30. Weidmer-Mikhail E, Sheldon S, Ghaziuddin M. Chromosomes in autism and related pervasive developmental disorders: a cytogenetic study. J Intellect Disabil Res 1998;42(Pt 1):8-12.

31. Castermans D, Wilquet V, Steyaert J, Van de Ven W, et al. Chromosomal anomalies in individuals with autism: a strategy towards the identification of genes involved in autism. Autism 2004;8(2):141-161.

32. Vorstman JA, Staal WG, van Daalen E, van Engeland H, et al. Identification of novel autism candidate regions through analysis of reported cytogenetic abnormalities associated with autism. Mol Psychiatry 2006;11(1):1, 18-28.

33. Zappella M. Autism and hypomelanosis of Ito in twins. Dev Med Child Neurol 1993;35(9):826-832.

34. Akefeldt A, Gillberg C. Hypomelanosis of Ito in three cases with autism and autisticlike conditions. Dev Med Child Neurol 1991;33(8):737-743.

35. Ballif BC, Rorem EA, Sundin K, Lincicum M, et al. Detection of low-level mosaicism by array CGH in routine diagnostic specimens. Am J Med Genet A 2006;140(24): 2757-2767.

36. Battaglia A, Bonaglia MC. The yield of subtelomeric FISH analysis in the evaluation of autistic spectrum disorders. Am J Med Genet C Semin Med Genet 2006;142(1):8-12.

37. Keller K, Williams C, Wharton P, Paulk M, et al. Routine cytogenetic and FISH studies for 17 p11/15q11 duplications and subtelomeric rearrangement studies 
in children with autism spectrum disorders. Am J Med Genet A 2003;117(2):105111.

38. Nair-Miranda K, Murch A, Petterson B, et al. An investigation into sub-telomeric deletions of chromosome 22 and pervasive developmental disorders. Am J Med Genet B Neuropsychiatr Genet 2004;125(1):99-104.

39. Wolff DJ, Clifton K, Karr C, Charles J. Pilot assessment of the subtelomeric regions of children with autism: detection of a 2q deletion. Genet Med 2002;4(1):10-14.

40. Knight SJ, Regan R, Nicod A, Horsley SW, et al. Subtle chromosomal rearrangements in children with unexplained mental retardation. Lancet 1999;354(9191): 1676-1681.

41. Jacquemont ML, Sanlaville D, Redon R, Raoul O, et al. Array-based comparative genomic hybridisation identifies high frequency of cryptic chromosomal rearrangements in patients with syndromic autism spectrum disorders. J Med Genet 2006; 43(11):843-849.

42. Schaefer GB, personal communication, 2007.

43. Clifford S, Dissanayake C, Bui QM, Huggins R, et al. Autism spectrum phenotype in males and females with fragile X full mutation and premutation. JAutism Dev Disord 2007;37(4):738-747.

44. Cohen IL, Sudhalter V, Pfadt A, Jenkins EC, et al. Why are autism and the fragile-X syndrome associated? Conceptual and methodological issues. Am J Hum Genet 1991;48(2):195-202.

45. Hatton DD, Sideris J, Skinner M, Mankowski J, et al. Autistic behavior in children with fragile X syndrome: prevalence, stability, and the impact of FMRP. Am J Med Genet A 2006;140(17):1804-1813.

46. Chudley AE, Gutierrez E, Jocelyn LJ, Chodirker BN. Outcomes of genetic evaluation in children with pervasive developmental disorder. J Dev Behav Pediatr 1998;19(5): 321-325.

47. Voigt RG, Dickerson CL, Reynolds AM, Childers DO, et al. Laboratory evaluation of children with autistic spectrum disorders: a guide for primary care pediatricians. Clin Pediatr (Phila) 2000;39(11):669-671.

48. Watson MS, Leckman JF, Annex B, Breg WR, et al. Fragile X in a survey of 75 autistic males. N Engl J Med 1984;310(22):1462.

49. Carney RM, Wolpert CM, Ravan SA, Shahbazian M, et al. Identification of MeCP2 mutations in a series of females with autistic disorder. Pediatr Neurol 2003;28(3): 205-211.

50. Davidovitch M, Patterson B, Gartside P. Head circumference measurements in children with autism. J Child Neurol 1996;11(5):389-393.
51. Fidler DJ, Bailey JN, Smalley SL. Macrocephaly in autism and other pervasive developmental disorders. Dev Med Child Neurol 2000;42(11):737-740.

52. Lainhart JE, Bigler ED, Bocian M, Coon H, et al. Head circumference and height in autism: a study by the Collaborative Program of Excellence in Autism. Am J Med Genet A 2006;140(21):2257-2274.

53. Lainhart JE, Piven J, Wzorek M, Landa R, et al. Macrocephaly in children and adults with autism. J Am Acad Child Adolesc Psychiatry 1997;36(2):282-290.

54. Butler MG, Dasouki MJ, Zhou XP, Talebizadeh Z, et al. Subset of individuals with autism spectrum disorders and extreme macrocephaly associated with germline PTEN tumour suppressor gene mutations. J Med Genet 2005;42(4):318-321.

55. Sikora DM, Pettit-Kekel K, Penfield J, Merkens LS, et al. The near universal presence of autism spectrum disorders in children with Smith-Lemli-Opitz syndrome. Am J Med Genet A 2006;140(14):1511-1518.

56. Tierney E, Bukelis I, Thompson RE, Ahmed K, et al. Abnormalities of cholesterol metabolism in autism spectrum disorders. Am J Med Genet B Neuropsychiatr Genet 2006;141(6):666-668.

57. GeneTests. Copyright University of Washington, Seattle, 1993-2007.

58. Baieli S, Pavone L, Meli C, Fiumara A, et al. Autism and phenylketonuria. J Autism Dev Disord 2003;33(2):201-204.

59. Chess S. Autism in children with congenital rubella. J Autism Child Schizophr 1971; 1(1):33-47.

60. Alsdorf R, Wyszynski DF. Teratogenicity of sodium valproate. Expert Opin Drug Saf 2005;4(2):345-353.

61. Arndt TL, Stodgell CJ, Rodier PM. The teratology of autism. Int J Dev Neurosci 2005;23(2-3):189-199.

62. Stromland K, Nordin V, Miller M, Akerstrom B, et al. Autism in thalidomide embryopathy: a population study. Dev Med Child Neurol 1994;36(4):351-356.

63. Sebat J, Lakshmi B, Malhotra D, Troge J, et al. Strong association of de novo copy number mutations with autism. Science 2007;316(5823):445-449.

64. Szatmari P, Paterson AD, Zwaigenbaum L, Roberts W, et al. Mapping autism risk loci using genetic linkage and chromosomal rearrangements. Nat Genet 2007;39(3): 319-328.

65. Jaenisch R, Bird A. Epigenetic regulation of gene expression: how the genome integrates intrinsic and environmental signals. Nat Genet 2003;33(Suppl):245254.

66. Lopez-Rangel E, Lewis ME. Further evidence for epigenetic influence of MECP2 in Rett, autism and Angelman's syndromes. Clin Genet 2006;69(1):23-25. 